\title{
Diagnóstico de saúde de um Município Paulista de pequeno porte: Dados preliminares
}

\section{Health diagnosis of a small city in São Paulo: Preliminary data}

DOI: $10.46814 / \operatorname{lajdv2n5-015}$

Recebimento dos originais: 10/07/2020

Aceitação para publicação: 30/08/2020

\author{
Carolina Beil \\ Médica pela Faculdade de Medicina de Ribeirão Preto - Universidade de São Paulo \\ Instituição: Universidade de São Paulo \\ Endereço: Av. Bandeirantes, 3900, Monte Alegre, Ribeirão Preto-SP, Brasil \\ E-mail: carolbeil14@hotmail.com \\ Carlos Augusto Rodrigues Padilha \\ Médico pela Faculdade de Medicina de Ribeirão Preto - Universidade de São Paulo \\ Instituição: Universidade de São Paulo \\ Endereço: Av. Bandeirantes, 3900, Monte Alegre, Ribeirão Preto-SP, Brasil \\ E-mail: rpadilha.carlos@gmail.com \\ Janise Braga Barros Ferreira \\ Doutor em Saúde Pública pela Universidade de São Paulo \\ Instituição: Universidade de São Paulo \\ Endereço: Av. Bandeirantes, 3900, Monte Alegre, Ribeirão Preto-SP, Brasil \\ E-mail: janise@fmrp.usp.br

\section{Luciana Cisoto Ribeiro} \\ Doutor em Ciências pela Universidade Federal de São Paulo. \\ Instituição: Universidade de São Paulo \\ Endereço: Av. Bandeirantes, 3900, Monte Alegre, Ribeirão Preto-SP, Brasil \\ E-mail: lcisoto@fmrp.usp.br

\section{Luciane Loures dos Santos}

Doutor em Ciências da Saúde pela Universidade de São Paulo/Faculdade de Medicina de Ribeirão

Preto/Departamento de Medicina Social

Instituição: Universidade de São Paulo

Endereço: Av. Bandeirantes, 3900, Monte Alegre, Ribeirão Preto-SP, Brasil

E-mail: luloures@fmrp.usp.br

\section{RESUMO}

A maioria dos municípios brasileiros (70\%) possui menos de 20.000 habitantes e apresentam semelhanças quanto às doenças mais prevalentes, características dos serviços e indicadores de saúde. Foi desenvolvido um estudo descritivo e retrospectivo em Cássia dos Coqueiros, um município de pequeno porte com características rurais no estado de São Paulo. Avaliou-se o perfil de morbimortalidade, cujas principais causas de internação foram gravidez, parto e puerpério, doenças cardiovasculares, causas externas, seguidas das doenças digestivas e respiratórias. As causas mais frequentes de mortalidade foram as doenças cardiovasculares, neoplasias, doenças respiratórias, 
causas externas e infecciosas. Estes achados contribuem para a compreensão da realidade sanitária de municípios rurais, suas potencialidades e debilidades, possibilitando a produção de informações acerca da situação sanitária e de gestão, as quais poderão subsidiar a construção de futuras políticas públicas do setor saúde.

Palavras chaves: Indicadores de Morbimortalidade, Saúde da População Rural, Cidades Pequenas, Redes de Atenção à Saúde.

\begin{abstract}
The majority of Brazilian municipalities (70\%) has fewer than 20.000 people and has similarities as the most prevalent diseases, typically services and health indicators. A descriptive and retrospective study was developed in Cássia dos Coqueiros, a small municipality with rural characteristics in the state of São Paulo. The morbidity and mortality profile was evaluated and the main causes of hospitalization were for pregnancy, childbirth and the puerperium cardiovascular, digestive and respiratory diseases, followed by external causes and the most frequent causes of death were cardiovascular diseases, cancers, respiratory diseases, external causes and infections. These findings contribute to the understand the health situation of rural municipalities, their strengths and weaknesses, enabling the production of information about the health status and management, which may support the construction of future public policies in the health sector.
\end{abstract}

Keywords: Indicators of Morbidity and Mortality, Rural health, Small cities, Health Care Networks.

\title{
1 INTRODUÇÃO
}

A maioria dos municípios brasileiros (70\%) possui menos de 20.000 habitantes e apresentam semelhanças quanto às doenças mais prevalentes, características dos serviços e indicadores de saúde. Realizar o diagnóstico de saúde de municípios pequenos onde a Saúde da Família está se consolidando é essencial para a organização do serviço de modo a privilegiar as áreas que mais necessitem. Pesquisas que busquem a compreensão dessa realidade sanitária abrem a possibilidade de produção de conhecimentos específicos que poderão subsidiar a construção de futuras políticas públicas de saúde.

\section{OBJETIVOS}

Identificar aspectos do diagnóstico de saúde em um município de pequeno porte com características rurais, a partir do perfil socioeconômico da população e as principais causas de morbimortalidade e suas possíveis implicações para a organização da assistência à saúde no município. 


\section{METODOLOGIA}

É um estudo descritivo, transversal e quantitativo onde foram analisados a faixa etária, sexo, analfabetismo, renda e situação sanitária, além das causas de morbimortalidade da população segundo faixa etária, sexo e principais agravos. Foram estudados os dados populacionais retrospectivos dos residentes de Cássia dos Coqueiros, um município de pequeno porte no interior do estado de São Paulo. Os dados estudados foram produzidos pelo Instituto Brasileiro de Geografia e Estatística (IBGE), disponibilizados no sítio eletrônico do Departamento de Informática do SUS (DATASUS) no período de 1999 à 2010. O projeto foi aprovado pelo Comitê de Ética em Pesquisa (Parecer $n^{\circ}$ 217.241)

\section{RESULTADOS}

Cássia dos Coqueiros tem 2.634 habitantes, 32\% residente na zona rural e 51\% de homens. O analfabetismo vem caindo (8\%), mas ainda é o dobro entre negros e pardos que nos brancos. A população de baixa renda também é maior entre negros $(54,3 \%)$ e pardos $(39,6 \%)$ e menor em brancos $(22,6 \%)$. As principais causas das internações foram gravidez, parto e puerpério $(14,9 \%)$, doenças cardiovasculares $(14,4 \%)$, causas externas $(11,8 \%)$, doenças digestivas $(10,4 \%)$ e respiratórias $(9,4 \%)$. As principais causas de mortalidade foram as doenças do aparelho circulatório $(25,6 \%)$, neoplasias $(18,1 \%)$, doenças respiratórias $(15,6 \%)$, causas externas $(9,4 \%)$ e infecciosas $(8,8 \%)$. E $80 \%$ dos acidentes de transporte envolveram homens com idade entre 20 e 59 anos.

\section{CONCLUSÃO}

Cássia dos Coqueiros é um município rural com perfil de cidade de grande porte, destacamos o elevado número de mortes de jovens por acidentes de trânsito e as desigualdades sociais que precisam ser consideradas no planejamento local para super as iniquidades. O conhecimento deste perfil auxilia na compreensão da realidade sanitária de municípios rurais, pois a partir de suas características, potencialidades e debilidades, abre-se a possibilidade de produção de informações específicas acerca da situação sanitária e de gestão, as quais poderão subsidiar a construção de futuras políticas públicas do setor saúde. 


\section{REFERENCIAS}

DATASUS. Departamento de informática do Sistema único de saúde (TABNET). Morbidade hospitalar do SUS - por local de residência - São Paulo. Internações por Caráter atendimento segundo Município - Cássia dos Coqueiros. 2014. Brasília, 2008. Disponível em:. http://www2.datasus.gov.br/DATASUS/index.php?area=0203\&id=6927\&VObj=http://tabnet.datasus .gov.br/cgi/deftohtm.exe?sih/cnv/nr. Acesso em: 20 set. 2020.

DATASUS. Departamento de informática do Sistema único de saúde. Informações de Saúde (TABNET). Brasília, DF, 2010. Disponível em: http://www2.datasus.gov.br/DATASUS/index.php. Acesso em: 20 set 2020.

IBGE - INSTITUTO BRASILEIRO DE GEOGRAFIA ESTATÍSTICA. Censo demográfico 2010: características da população e dos domicílios: resultados do universo. Rio de Janeiro, 2011. Disponível em: <http://www.ibge.gov.br/english/estatistica/populacao/censo2010/caracteristicas_da_populacao/result ados_do_universo.pdf>. Acesso em: 20 set 2020.

IBGE - INSTITUTO BRASILEIRO DE GEOGRAFIA E ESTATÍSTICA. IBGE Cidades@. Cássia dos Coqueiros. Rio de Janeiro, $2012 . \quad$ Disponível em: http://www.cidades.ibge.gov.br/xtras/perfil.php?lang=\&codmun=351090\&search=sao-paulo|cassiados-coqueiros|infograficos:-informacoes-completas. Acesso em: 20 set. 2020.

PEEK-ASA, C.; ZWERLING, C.; STALLONES, L. Acute traumatic injuries in rural populations. American Journal of Public Health, Boston, v. 94, n.10 p.1689-1693, oct. 2004. 